\title{
Orts- und Flurwüstungen in Brail (bei Zernez)
}

Peter Abt

Vielerorts beobachtet man Wandlungen der alpinen Kulturlandschaft ${ }^{1}$. Auch die Fraktion Brail bei Zernez wird sich in den nächsten Jahren weiter verändern. Wohl die auffälligsten Anzeichen dafür sind einige neuerstellte Ferienhäuser oberhalb des Dörfchens. Im folgenden möchten wir auf einige Wüstungserscheinungen und Funktionswandlungen hinweisen.

\section{Ackerterrassen}

Deutlich sichtbare Relikte eines ehemals ausgedehnten Ackerbaus sind die Terrassen. Gutersohn

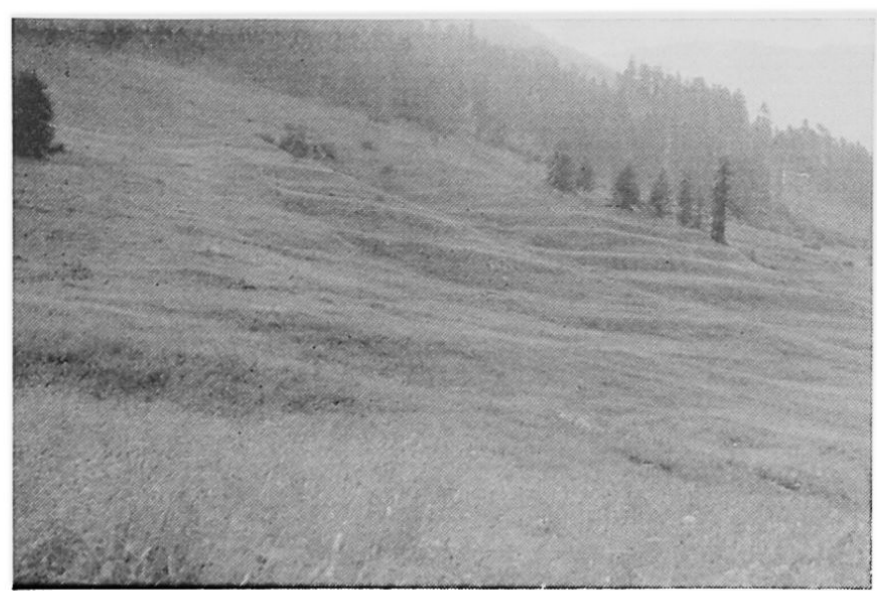

Abbildung 1. Ackerterrassen oberhalb Brail in der Flur Gundscheras, Höhe etwa 1750 m

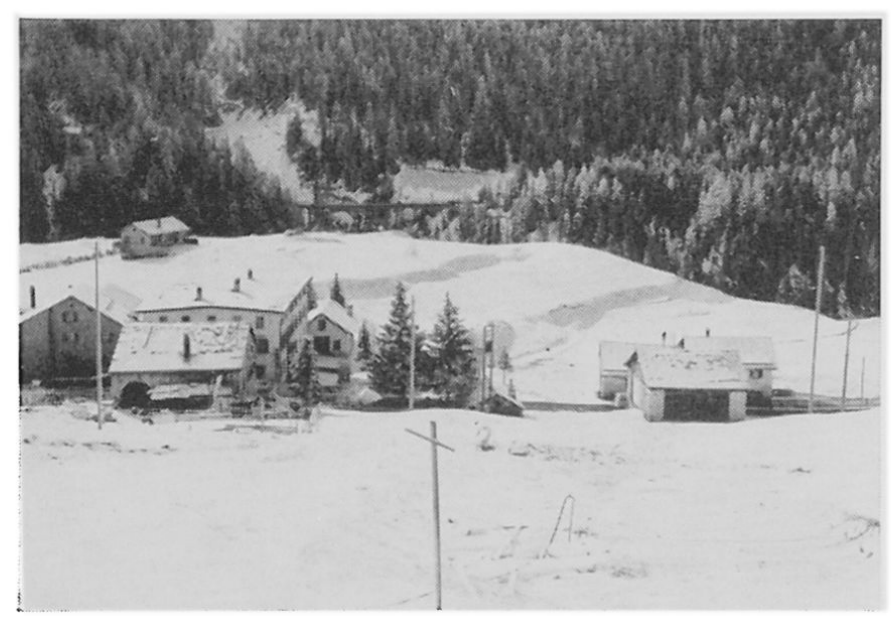

Abbildung 2. Ackerterrassen unterhalb Brail in der Flur Craistas, Höhe etwa 1630 m. Die Ziffer 2 markiert das Backhaus schreibt über das Unterengadin?: "Noch vor 200 Jahren war die Selbstversorgung dank der vielen terrassierten Äcker der sonnigen Hänge mehr als gesichert. Bei Ramosch z. B. lagen bis zu 30 Terrassen übereinander. Schon um 1800 war die Ackerflur gegenüber dem 17. Jahrhundert als Folge starker Emigration zurückgegangen, aber noch zu Anfang des 19. Jahrhunderts war Selbstversorgung mit Brotgetreide üblich, und es blieb sogar noch ein Überschuß für den Verkauf nach auswärts, zum Beispiel ins Oberengadin, übrig.»

In Brail beobachten wir Ackerterrassen oberhalb des Dorfes in den Fluren Muschnuns und Gundscheras in einer Höhenlage von 1630 bis 1770 m (Abb. 1,

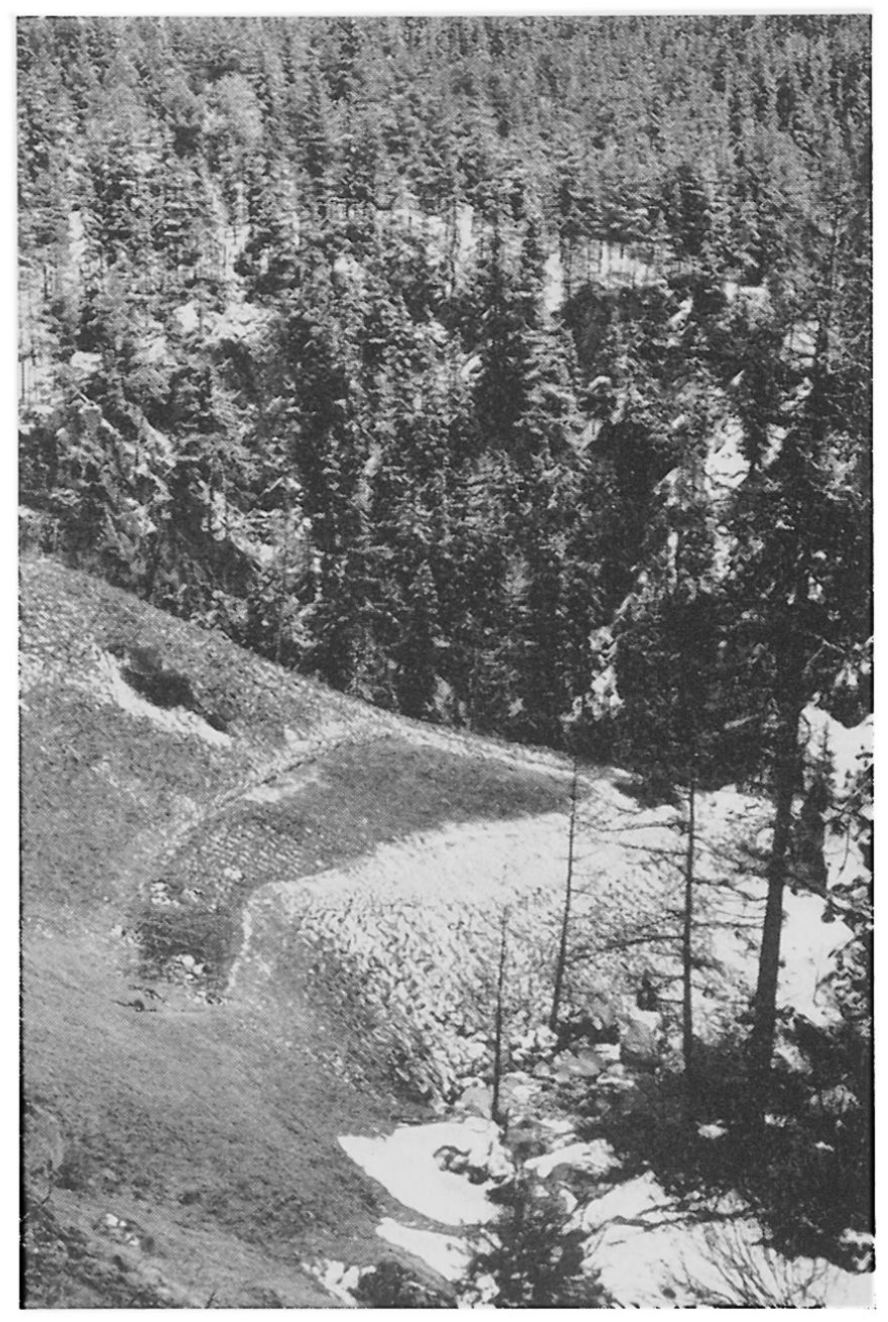

Abbildung 3. Acker im Val Punt Ota. 1969 wurde noch die Hälfte davon mit Kartoffeln und Gemüse bestellt 


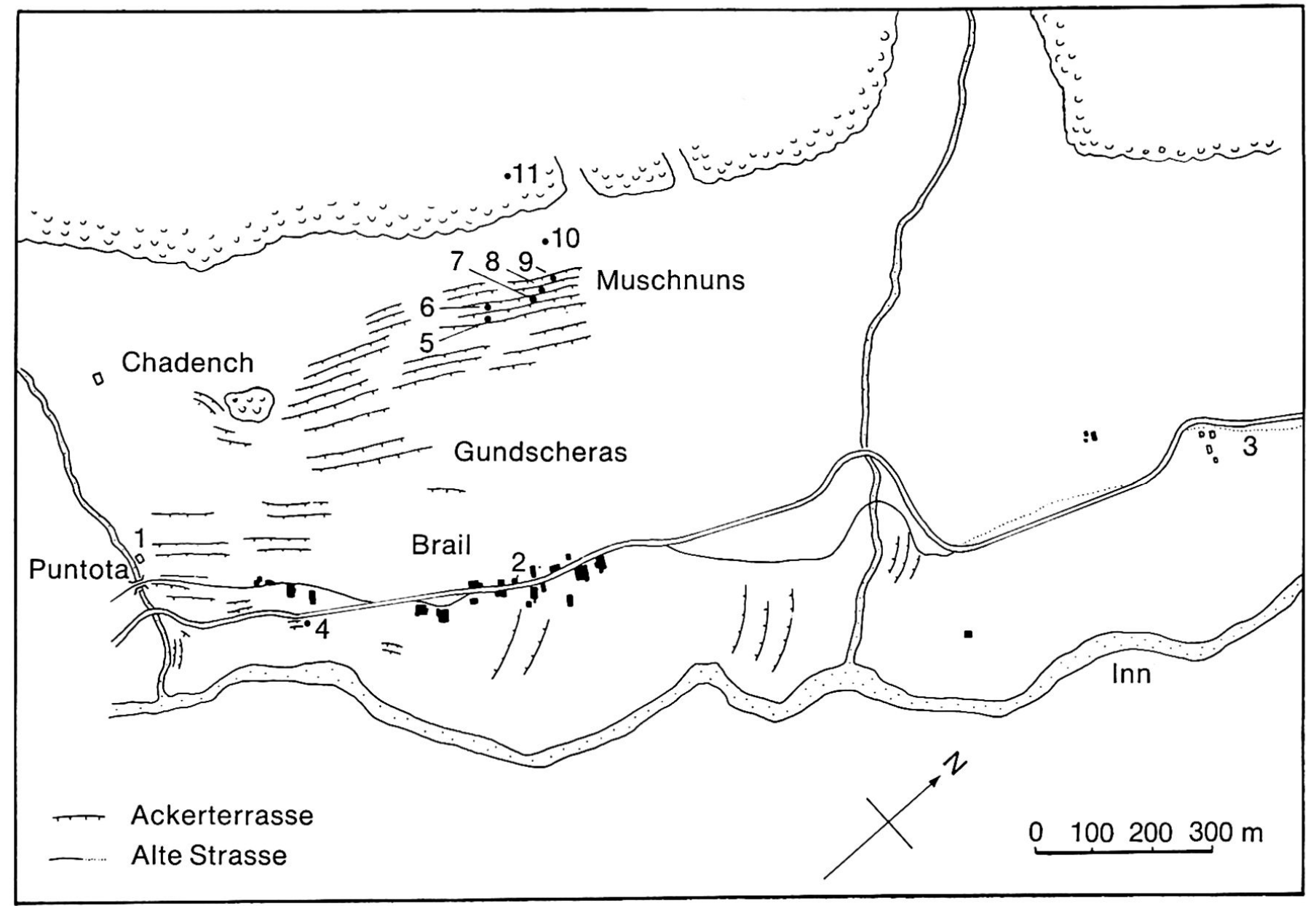

Figur 1

Fig. 1). Hier wurde besonders Gerste angebaut. Aber auch unterhalb des Dorfes gegen den Inn liegen aufgelassene Äcker (Abb. 2), vorzugsweise in den geschützten Mulden der Seitenbäche (Val Punt Ota, Abb. 3, Val Stretta, Val Barlas-ch). Diese Lagen eignen sich gut für den Anbau von Kartoffeln. Die ehemaligen Ackerflächen lassen sich durch Phosphatanalysen nachweisen. Mit der Tüpfelmethode nach Gundlach ${ }^{3}$ ermittelten wir:

Fig. 1

Nr.

4 Acker, 1969 mit Kartoffeln bestellt

5 ehem. Acker, 1969 als Wiese genutzt

6 ehem. Acker, 1969 als Wiese genutzt

7 ehem. Acker, 1969 als Wiese genutzt

8 ehem. Acker, 1969 als Wiese genutzt

9 ehem. Acker, 1969 als Wiese genutzt

10 Weide

11 Wald

Die höhergelegenen Äcker (Proben Nr. 8, 9) sind offenbar weniger stark gedüngt oder früher aufgegeben worden. Die untenstehende Tabelle 4 gilt zwar für die ganze Gemeinde Zernez, doch wird auch für Brail der Zweite Weltkrieg eine letzte merkliche Ausdehnung des Ackerlandes gebracht haben. Zwi- schen 1945 und 1950 erfolgte die Güterzusammenlegung. Damit kam der Ackerbau praktisch vollständig zum Erliegen. Die Luftaufnahme (Abb. 4) von 1961 zeigt noch etwa 9 Äckerlein, 1969 wurden durch Feldbegehung 5 festgestellt ${ }^{5}$.

In seiner Dissertation äußert H. Schmid 1955 die Meinung, «daß Ackerbau auch im Engadin mit Erfolg gepflegt werden kann und gepflegt werden sollte ${ }^{6} . »$ Er bezieht sich dabei auf die Versuche, welche unter der Kontrolle der Bündnerischen Pflanzenbaukommission in La Punt durchgeführt wurden und welche erstaunlich hohe Hektarerträge ergaben. Schmid meint, daß die schlechten Ergebnisse nicht nur auf die ungünstige Witterung, sondern auch auf verschwundene Erfahrungen und Kenntnisse des Ackerbaus zurückzuführen seien. 1964 wird in der Schrift «Die Landwirtschaft Graubündens, eine Studie für die landwirtschaftliche Betriebsberatung» 7 die Auffassung vertreten (für den ganzen Kanton): "Während klimatisch eine Ausdehnung des Ackerlandes möglich wäre, verbietet der hohe Arbeitsaufwand im unebenen Gebiet die Entwicklung dieser Nutzung; sie wird der Arbeitsrationalisierung wegen eher zurückgehen. Eine Ausnahme macht der Anbau von Saatkartoffeln in gesunden Berglagen. Hier lohnt sich der höhere Aufwand.» Den Brailer Bauern fehlt es nicht grundsätzlich an der Erfahrung, eher an Arbeitskräften. Auch 


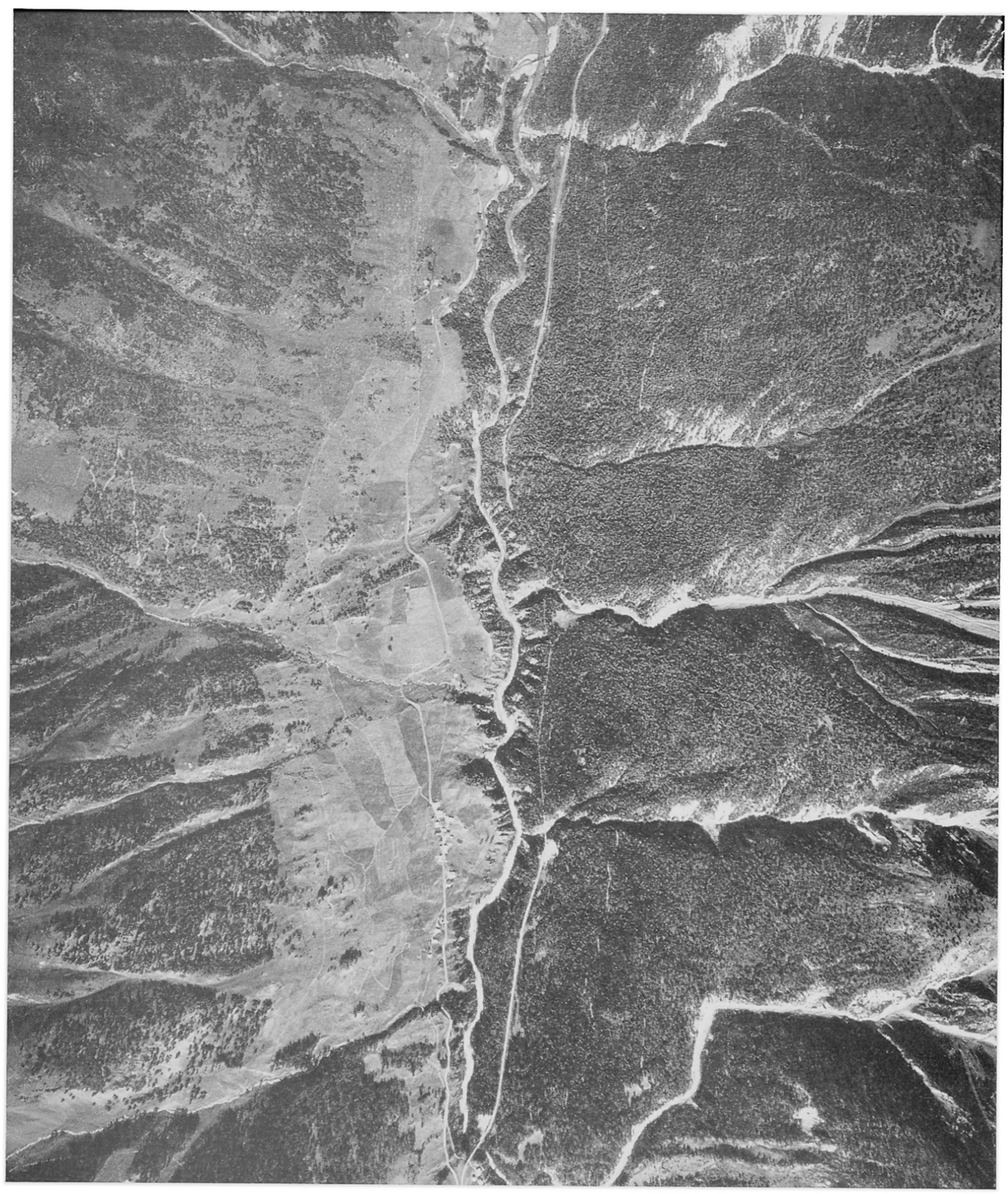

Abbildung 4. Flugaufnahme von Brail (Aufnahme der Eidgenössischen Landestopographie, Aufnahmedatum 29. 8. 61, Bewilligung zur Reproduktion vom 28. 8.69)

brachte die Güterzusammenlegung mit sich, daß einige Landwirte ihre bevorzugten Ackerparzellen gegen für diese Nutzung ungünstigeres Land eintauschten und damit den Ackerbau aufgaben. Nicht nur der Ackerbau, sondern die Landwirtschaft ganz allgemein geht in Brail zurück. Die jungen Leute wollen nicht mehr Bauern werden und wandern ab.
Die Überalterung des Dörfchens äußert sich etwa in der Schließung der Schule, die wenigen Kinder gehen heute nach Zernez. So ist es nicht erstaunlich, wenn gerade am Sonnenhang Land verkauft wird zur Erstellung von Ferienhäusern, welche nun in das ehemalige Ackerbaugebiet zu stehen kommen. 
Flächen in ha, 0 bedeutet weniger als 0,5 ha

$$
1917 \quad 1926 \quad 1942
$$

$\begin{array}{lrrr}\text { Winterweizen } & - & - & 0,09 \\ \text { Sommerweizen } & - & - & 0,68 \\ \text { Wintergerste } & - & - & - \\ \text { Sommergerste } & 10,8 & 13,3 & 12,27 \\ \text { Hafer } & 0,5 & 0,3 & 0,46 \\ \text { Roggen } & 0,8 & - & 0,65 \\ \text { Kartoffeln } & 5,4 & 2,6 & 10,54 \\ \text { Gemüse } & 0,08 & - & 0,89\end{array}$

$\begin{array}{rrrrrr}-7 & - & 0 & - & - & - \\ -18 & - & - & 1 & - & - \\ 18 & 18 & 18 & 14 & 13,41 & 10 \\ 3 & 1 & 4 & 2 & 2,04 & 4 \\ 1 & 2 & 1 & - & - & - \\ 11 & 9 & 8 & 6 & 4,05 & 2 \\ 2 & 1 & 0 & 0 & 0,83 & 0\end{array}$

\section{Wiistgelegte Mühle}

Die einzige Mühle von Brail lag unmittelbar oberhalb der Punt Ota («Hohe Brücke»; LK $1: 25$ 000, Blatt 1238, P. Quattervals, Koord. 798.175/169. 850, Fig. 1, Nr. 1). Erhaltengeblieben sind einzig etwas Mauerwerk (Abb. 5, 6) und zwei Mühlsteine. Der «Bodenstein» aus Augengneis befindet sich nun im Garten des Hotels Riveroni (Abb. 7), der obere Stein, aus stark gefälteltem Gneis, lehnt vor dem Haus neben der Kirche. Über den Zeitpunkt der Wüstlegung konnte nichts in Erfahrung gebracht

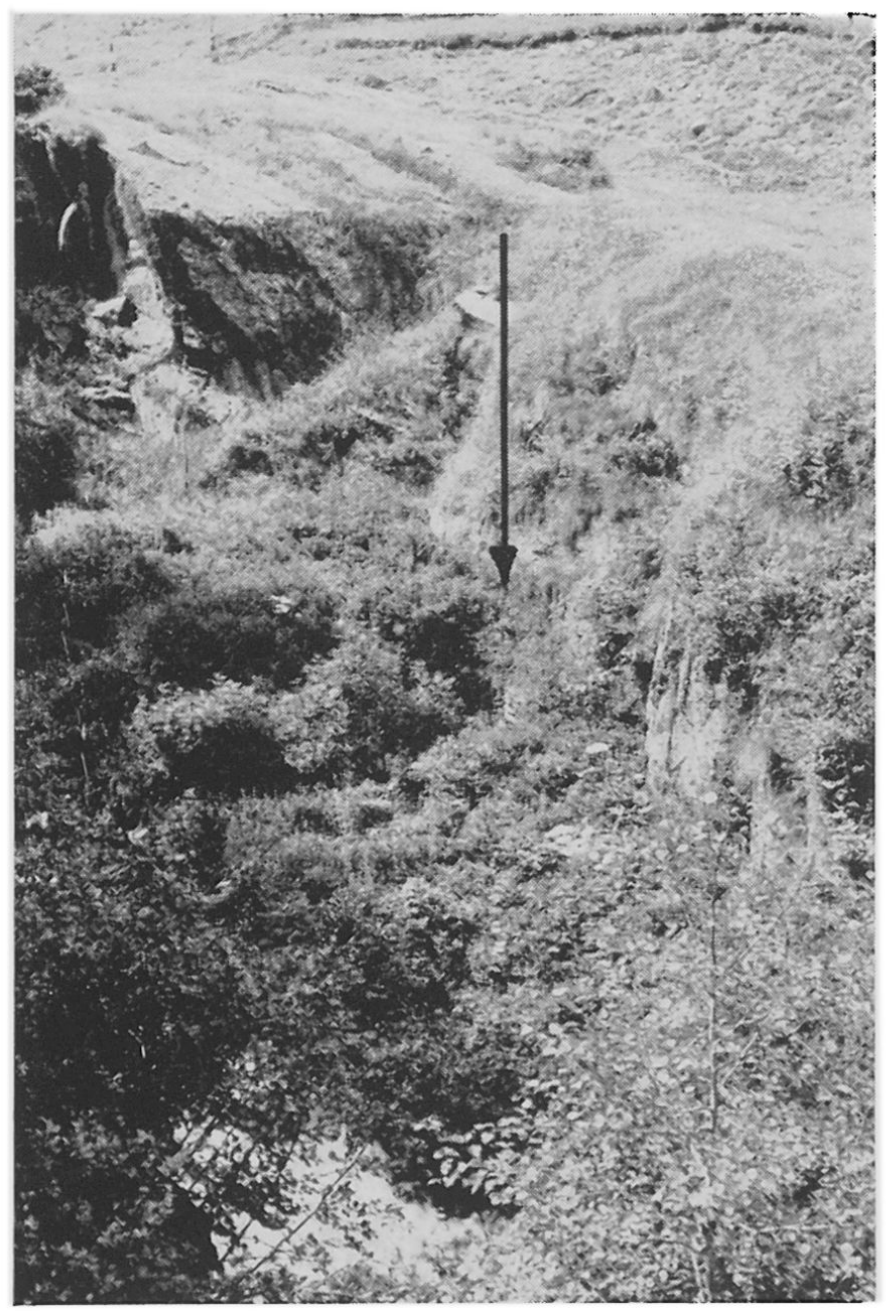

Abbildung 5. Der Pfeil deutet auf die Ruinen der Mühle im Val Punt Ota werden. Der Standort der Mühle im Val Punt Ota war insofern günstig, als hier eine bedeutend geringere Lawinengefahr herrschte als etwa im Val Barlas-ch.

\section{Backofen, Backhaus}

Früher besaßen 14 Haushaltungen einen eigenen Backofen. Besonders in der Zeit nach dem Zweiten Weltkrieg erneuerte man die Kücheneinrichtungen und brach bei dieser Gelegenheit die Öfen ab, so

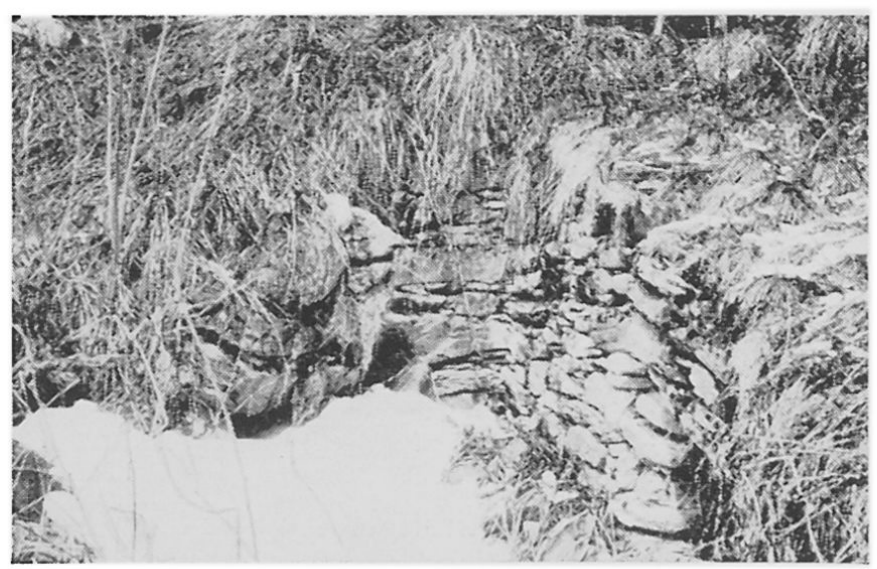

Abbildung 6. Im Spätfrühling sind die Mauerreste der Mühle deutlich zu erkennen

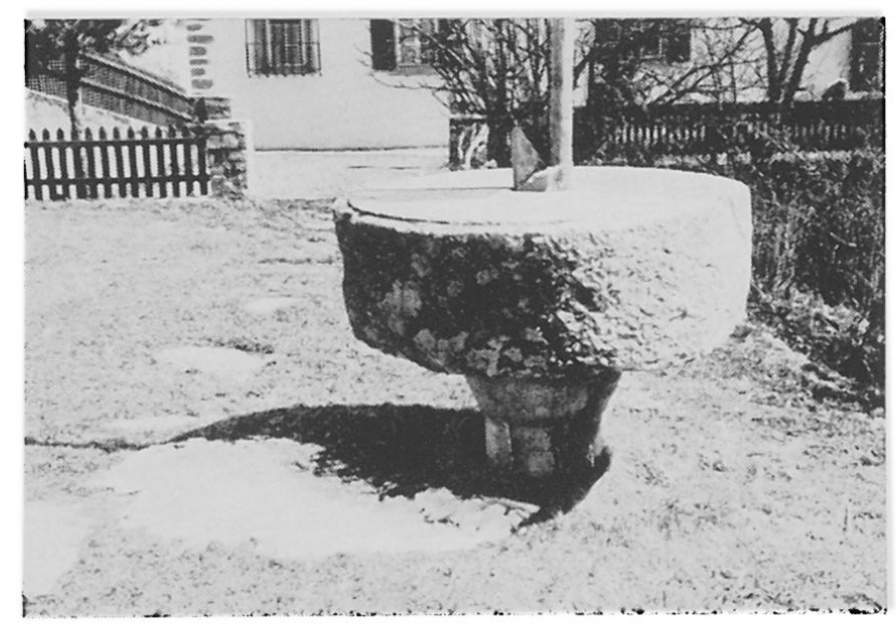

Abbildung 7. Unterer Mühlstein im Garten des Hotels Biveroni, Brail 


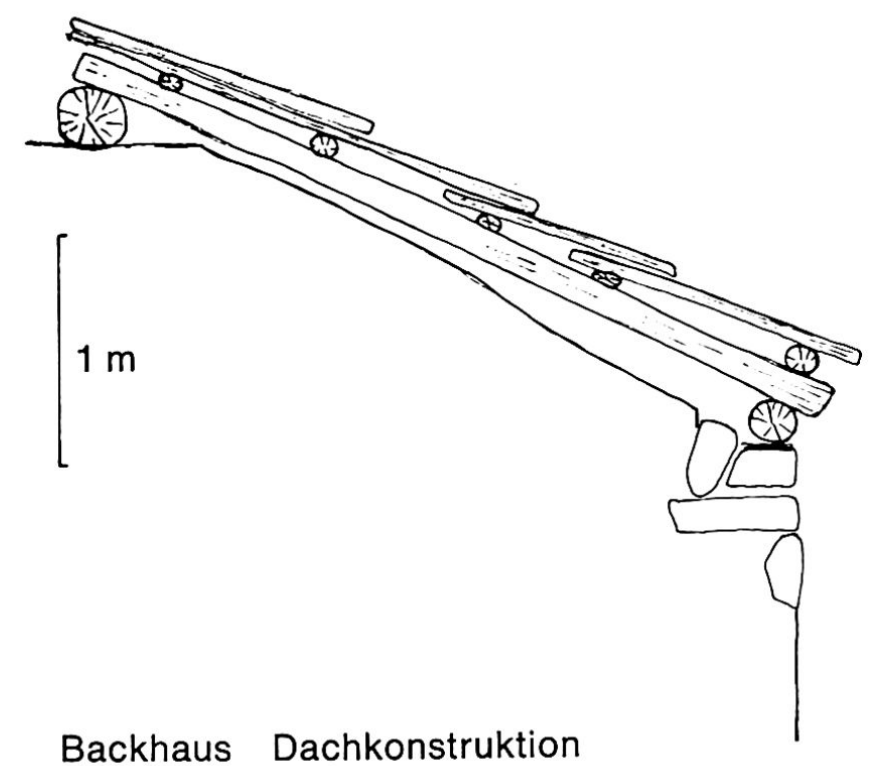

Fig. 2

daß heute nur noch einer übriggeblieben ist. Dieser befindet sich im Haus der Familie Waser (Abb. 8). Es handelt sich dabei um eine aus der Wand herausragende rechteckige Konstruktion, welche von der Küche aus bedient wurde. Der Ofen war bis in die Zeit von 1918 in Gebrauch.

Neben dem Hotel Post steht ein Backhaus (Fig. 1 Nr. 2, Abb. 2, 9, Fig. 2, 3, 4), das bis etwa 1910 benützt wurde. Der Ofenteil diente später noch als Militärküche, heute wird er nicht mehr gebraucht. Den Nebenraum dagegen baute man zum Autoeinstellplatz um. Während der Backofen recht gut erhalten blieb, zerfällt das Holzdach, auch das Mauerwerk und der Kamin sind stellenweise schadhaft.

\section{Wüstgelegte Höfe}

In der Umgebung von Brail deuten vier Flurnamen auf wüstgelegte Höfe hin ( Cha» = Haus):

Chadench 798.850/170.150 nw davon: God Chadench

Chaveglia 799.050/170.800

Chanoscha 799.050/171.600

Chafuatscha 799.300/171.650

außerdem stehen Ruinen bei Prazet 799.700/

172.100

\section{Chadench}

In einer flachen Mulde westlich der Kirche (797. 900/170.025/1750 m) beobachtet man stark zerfallendes Gemäuer (Abb. 10), welches einen rechteckigen Grundriß bildet. Wenngleich der Phosphatgehalt der Umgebung nicht besonders hoch ist Skalenwert $1-$ muß es sich hier um den wüstgelegten Hof Chadench handeln. Nirgendswo in der
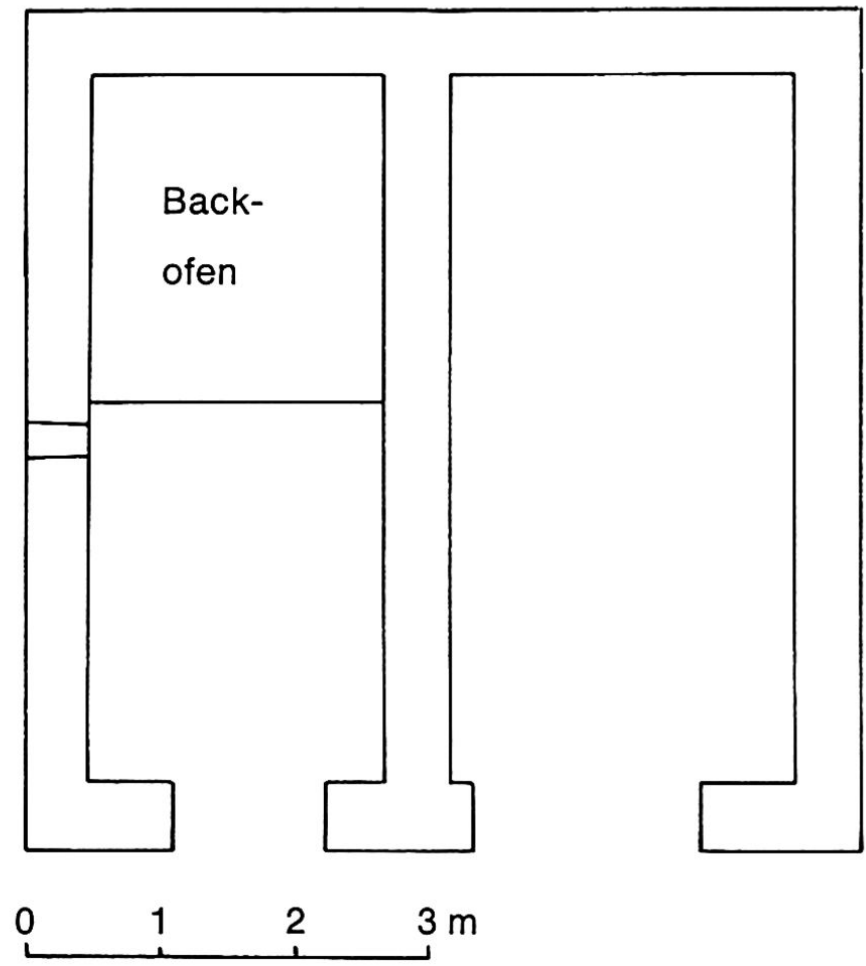

Grundriss Backhaus

Fig. 3

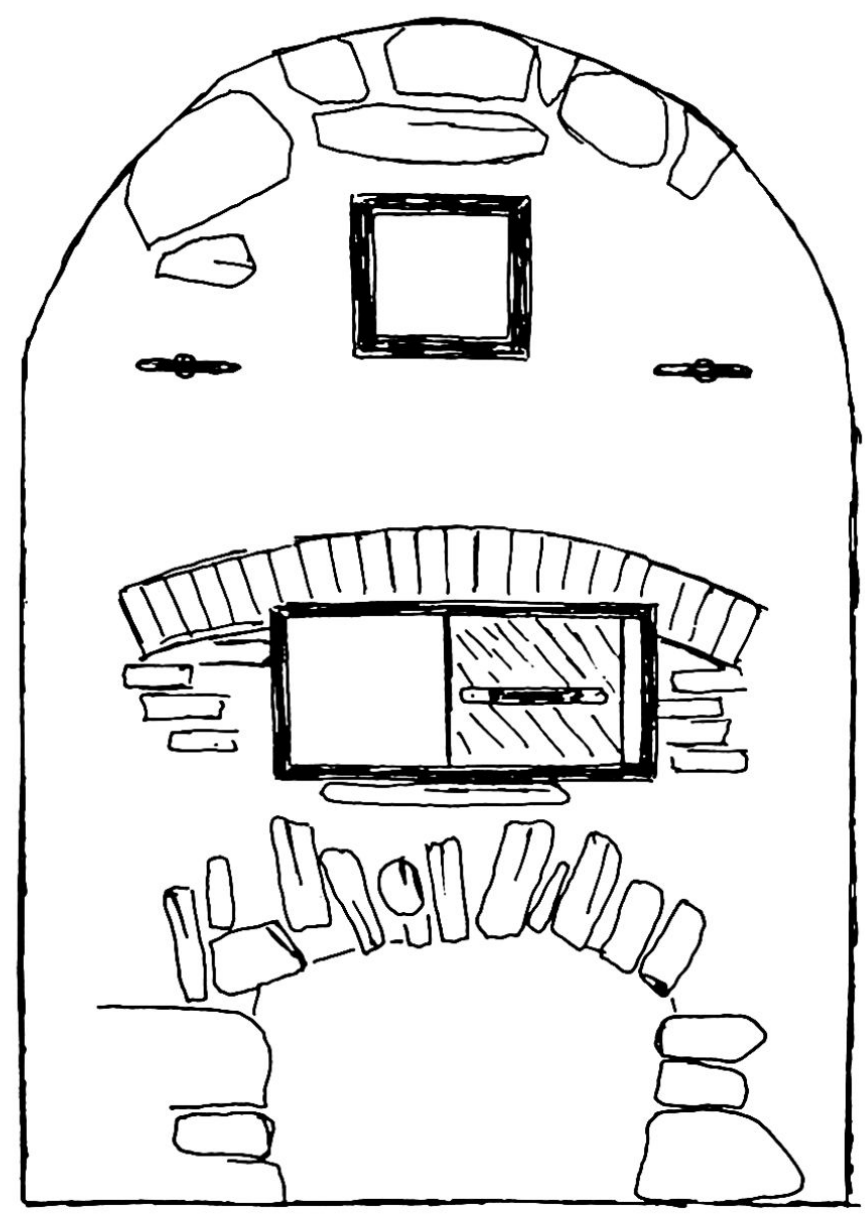

Backofen

Fig. 4 


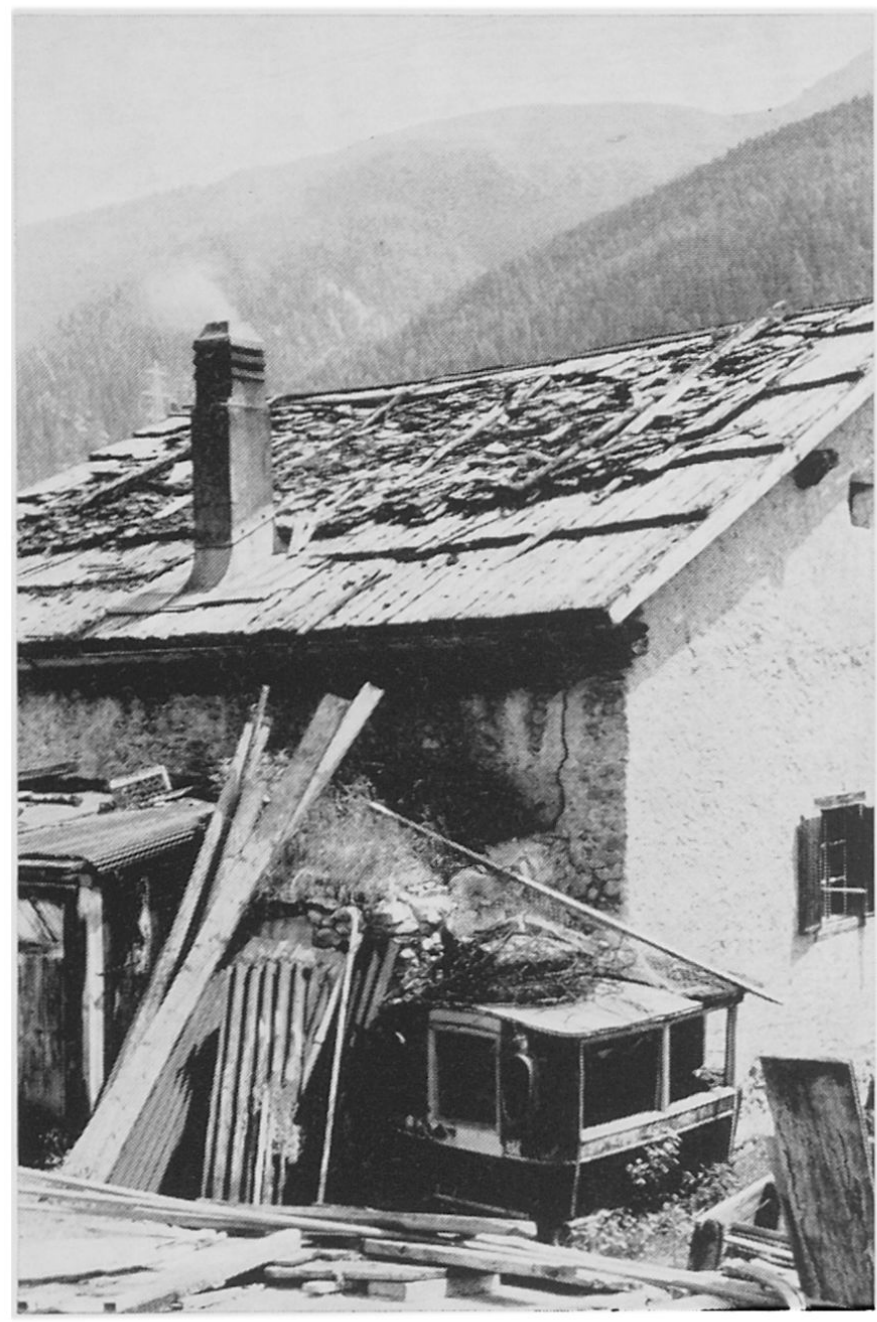

Abbildung 8. Kaum sichtbar ist der einzige noch erhaltene, aus der Wand herausragende Backofen (Haus Familie Waser)

gleichnamigen Flur konnten weitere Ruinen gefunden werden. Zeitpunkt und Ursache der Wüstlegung sind unbekannt.

\section{Chaveglia}

Im Gegensatz zu den übrigen Hofwüstungen sind den Einheimischen von Brail keine sichtbaren Überreste in der Flur Chaveglia bekannt. Eine Feldbegehung bestätigte diese Aussage.

\section{Chanoscha}

Auf einem Sporn unmittelbar östl. der Hauptstraße (799.250/171.375/1610 m, Fig. 1, Nr. 3), zeugt guterhaltenes Mauerwerk von einer ehemaligen Ansiedlung (Abb. 11). Auch hier ist der Phosphatgehalt an der Oberfläche niedrig (Skalenwert 1). Möglicherweise liegen die zu den Ruinen gehörenden Kulturschichten bedeutend tiefer. Wir möchten diesen Wüstungsplatz mit dem Flurnamen Chanoscha, der auf der Karte etwas nördlicher steht, in Zusammenhang bringen.

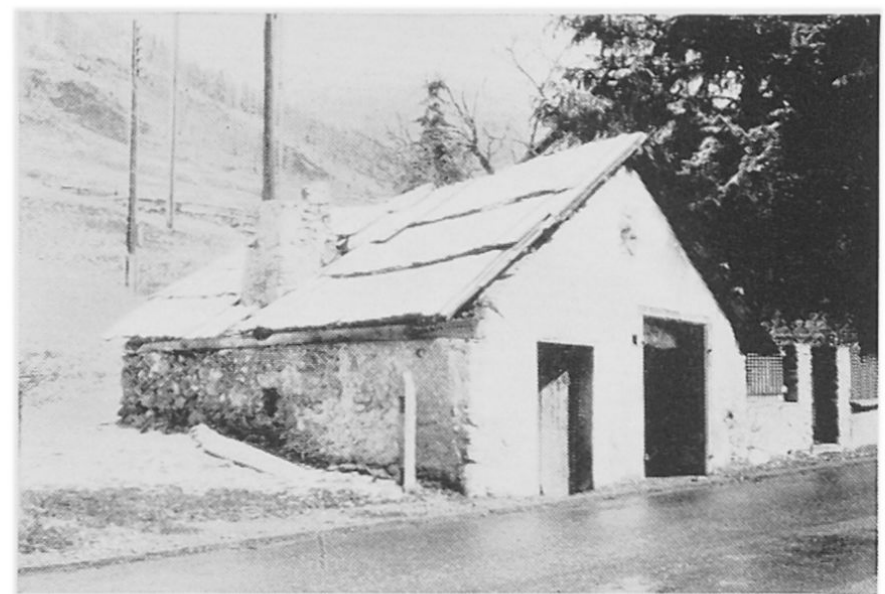

Abbildung 9. Das Backhaus von Brail

\section{Chafuatscha}

Bei den Mauerresten zwischen der alten Landstraße und der heutigen Hauptstraße (799.700/171.900/ $1605 \mathrm{~m}$ ) handelt es sich vermutlich um den aufgelassenen Hof Chafuatscha (Abb. 12), der entsprechende Flurname ist allerdings auf der Landeskarte etwas weiter südwestlich eingetragen. Heute sind noch die Grundrisse zweier Gebäude erkennbar. Chafuatscha wird in der Arbeit von Prof. G. C. Muoth über das «Statut del onorat comün da Zernez» 8 erwähnt. Muoth weist auf ein Schiedsgerichtsurteil hin, welches am 7. November 1571 in Zernez gefällt wurde. Die Bewohner von Chafuatscha und weiterer Höfe verlangten damals, weiterhin ganzjährlich dort zu wohnen und als Nachbarschaft mit eigener Weide und Alprechten anerkannt zu werden. Sie stützten sich dabei auf Dokumente von 1539 und 1547, doch das Schiedsgericht erklärte diese fortan als ungültig: offensichtlich war den Zernezern die Neubildung von Fraktionen höchst unwillkommen. In der Folge wurden die Höfe zu Maiensäßen, die man im Laufe der Zeit immer mehr vernachlässigte.

\section{Prazet}

Von den auf der Landeskarte eingetragenen zwei Gebäuden westlich des Dorfes Prazet (799.700/172. $100 / 1620 \mathrm{~m}$ ) stehen zurzeit nur noch Ruinen (Abb. 13). Das Gebäude mit der relativ gut erhaltenen talwärtsgerichteten Wand läßt sich mit Hilfe eines Türbalkens mit Jahreszahl auf 1614 datieren.

\section{Schlußbemerkung}

Am Beispiel von Brail versuchten wir aufzuzeigen, daß im Engadin immer noch Ackerbaurelikte anzutreffen sind. Mit der Zeit werden diese aber spärlicher werden. Das Interesse der einheimischen Bevölkerung an derartigen Dingen ist nicht überall ausgeprägt. Einige Bewohner von Brail waren z. B. höchst erstaunt, von einem Backhaus zu erfahren, 


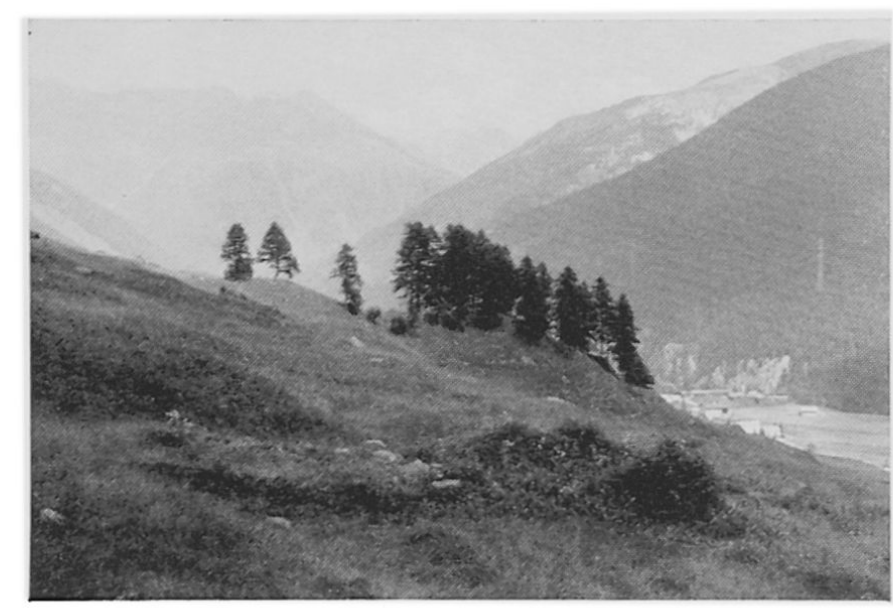

Abbildung 10. Die Wüstungsstelle des Hofes Chadench

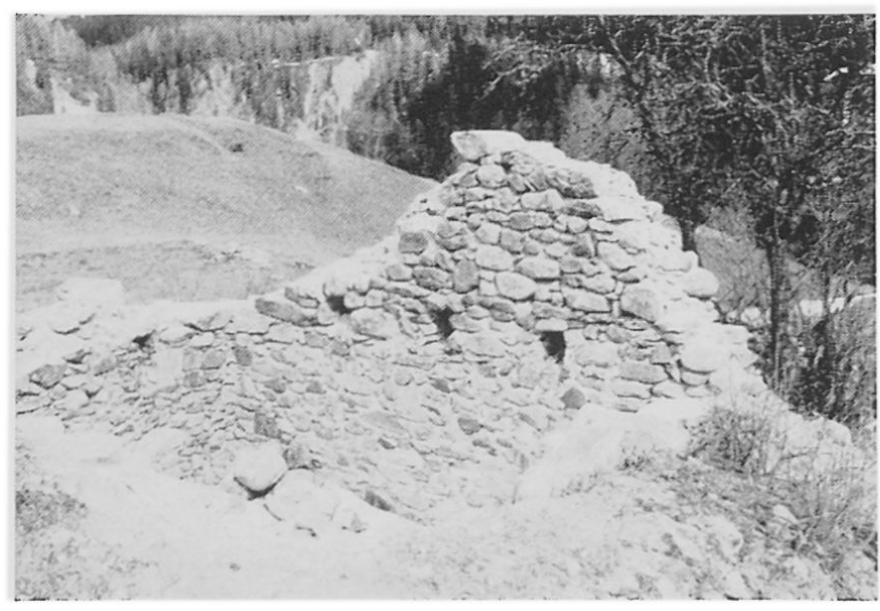

Abbildung 11. Chanoscha

obwohl sie tagtäglich mehrmals daran vorbeigingen. Allfällige weitere kulturlandschaftsgeschichtliche Arbeiten sollten daher möglichst rasch an die Hand genommen werden.

\section{Anmerkungen}

1 In der "Geographica Helvetica» erschienene Arbeiten: Gutersohn, H., 1964: Der gegenwärtige Wandel alpiner Kulturlandschaften, XIX, Nr. 3, 138-147.

Schwabe, E:. 1964: Alpine Erholungslandschaft, XIX, Nr. 3, S. 147-153.

Haefner, H., 1964: Der Strukturwandel der Alpwirtschaft im Luftbild (am Beispiel des Dischmatales) XIX, Nr. 4, S. 218-228.

Nigg, W. 1965: Landschaftliche Wandlungen im nördlichen Churer Rheintal, XX, Heft 3, S. 123 132.

Kilchenmann, A., 1967: Kulturgeographischer Strukturwandel auf der Lenzerheide, XXII, Heft 4, S. 258-261.

Bär, O., 1969: Chandolin 1968 - das Verschwinden der Alpwirtschaft aus einem Walliser Dorf, XXIV, Heft 1, S. 1-7.

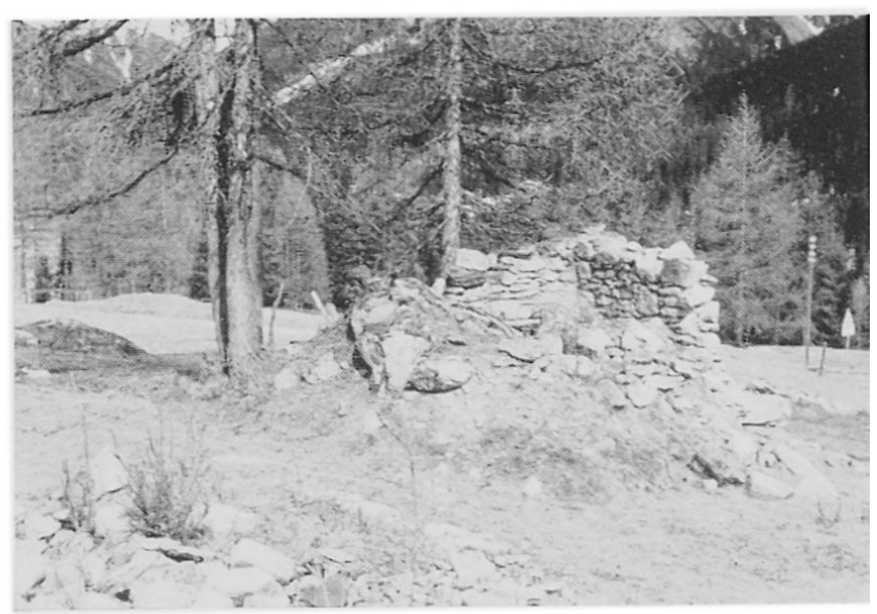

Abbildung 12. Chafuatscha

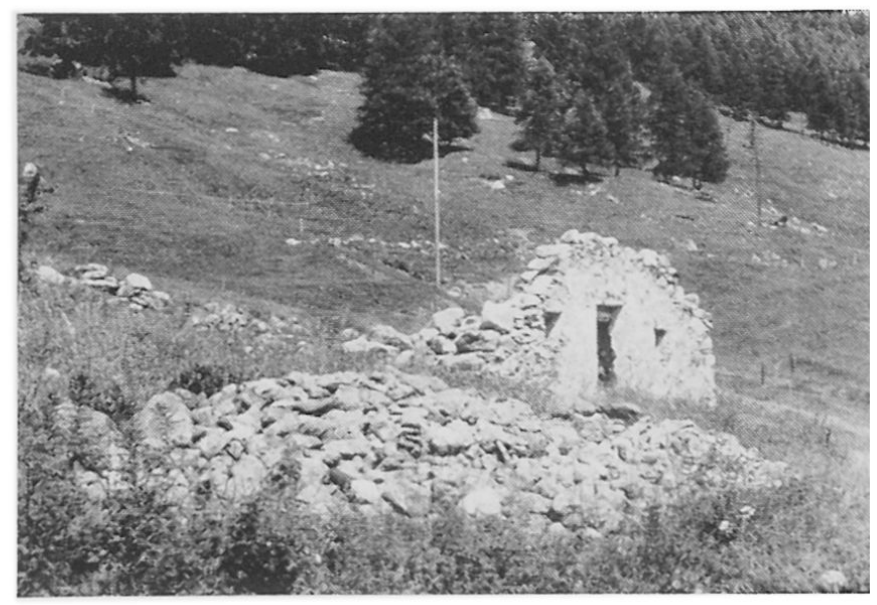

Abbildung 13. Ruinen in der Nähe des Hofes Praze

2 Gutersohn, H., 1961: Geographie der Schweiz Band II: Alpen, 1. Teil, S. 293.

3 Abt, P., 1969: Phosphatuntersuchungen zur topo. graphischen Lokalisation von Ortswüstungen «Geo graphica Helvetica», XXIV, Heft 4, S. 185-190.

4 Zusammengestellt aus den Veröffentlichungen de: Eidgenössischen Statistischen Amtes (Stat. Quellen werke der Schweiz).

5 1969: Kartoffeläcker: $48 \mathrm{~m}^{2}, 60 \mathrm{~m}^{2}, 45 \mathrm{~m}^{2}=$ Total $183 \mathrm{~m}^{2}$. Speisekarotten $175 \mathrm{~m}^{2}$ (1 Acker) diverse Gemüse $60 \mathrm{~m}^{2}$ (1 Acker).

6 Schmid, H., 1955: Die Oberengadiner Land- unc Alpwirtschaft, Zürcher Dissertation, S. 53.

7 Herausgegeben von der Dokumentationsstelle de SVBL, Zentralstelle Küsnacht, Post Erlenbach ZH Juli 1964, S. 16.

8 Muoth, G. C., 1898: Statut del onorat comür da Zernez (Segond la compilaziun e copia del 1724 scritta da Chaspar Jousch, da quel temp minister : Zernez) in: Annalas della Societad Rhaeto-roman scha, XII, Cuira, S. 117-119.

Für die Übersetzung dieses Textes bin ich Herrı Prof. Dr. Th. Ebneter, Zürich, zu großem Dank ver pflichtet. 